\title{
Overcoming Limitation in the Design of Selective Solid Catalysts by Manipulating Shape and Size of Catalytic Particles: Epoxidation Reactions on Silver
}

\author{
Suljo Linic* and Phillip Christopher ${ }^{[a]}$
}

Conventional solid metal catalysts, usually synthesized using wet impregnation strategies, are almost always quasi-spherical particles mainly terminated by low-energy surface facets, such as the (111) surface for fcc crystals and the (0001) surface for hcp crystals, and supported on high surface area materials. ${ }^{[1-3]}$ The diameter of these metal particles is from a few tens to a few hundreds of nanometers. The particle shapes and sizes represent a reasonable compromise between simple and scalable synthesis, relatively high surface to volume ratio, and high resistance to sintering and degradation under reaction conditions. A major negative consequence of the lack of diversity in the size and shape of metallic catalytic particles is that it has been difficult to identify and optimize selective catalysts for the production of reactive, commodity chemicals. These difficulties stem from large differences in the chemical activity of different metal elements. For example, identical surface sites of neighboring metals in the periodic table of elements bind various adsorbates with differences in adsorption energies of up to about $1 \mathrm{eV}$ (ca. $100 \mathrm{~kJ} \mathrm{~mol}^{-1}$ ), which means that whereas one metal might be chemically inert for a particular chemical transformation, its first neighbor in the periodic table might be overly reactive and equally inefficient. ${ }^{[4]}$

The above-discussed obstacles have prevented the design of selective solid catalysts for direct partial oxidation of propylene to propylene oxide ( $\mathrm{PO} ; \mathrm{C}_{3} \mathrm{H}_{6}+1 / 2 \mathrm{O}_{2} \rightarrow \mathrm{C}_{3} \mathrm{H}_{6} \mathrm{O}$ ). $\mathrm{PO}$ is used as a chemical intermediate in the production of foams, coatings, adhesives, propylene glycol, and glycol ethers. It has a market on the order of several billion dollars per year. PO is currently produced in costly homogeneous processes which require expensive post-reaction separation and use environmentally harmful oxidation reagents, mainly chlorohydrins or hydrogen peroxide, instead of air or oxygen. Direct heterogeneous processes are attractive because of the easy separation, long lifetime, and regenerability of solid catalysts, and the environmentally benign and cheap oxidizing agents (molecular $\mathrm{O}_{2}$ ). Conventional epoxidation catalysts containing silver particles dispersed on $\alpha$-alumina support, used in the analogous catalytic epoxidation of ethylene to form ethylene oxide $\left(\mathrm{C}_{2} \mathrm{H}_{4}+1 / 2 \mathrm{O}_{2} \rightarrow \mathrm{C}_{2} \mathrm{H}_{4} \mathrm{O}\right)$, are not selective since they activate allylic hydrogen in propylene, leading to complete combustion. In

\footnotetext{
[a] Prof. S. Linic, P. Christopher

University of Michigan, Department of Chemical Engineering

Ann Arbor, MI 48109-2136 (USA)

Fax: $(+1) 734-764-7453$

E-mail: linic@umich.edu
}

contrast, less chemically reactive gold-based catalysts do not activate $\mathrm{O}_{2}$ efficiently without sacrificial agents such as $\mathrm{H}_{2}$ and are therefore limited by low activity. Development of a robust heterogeneous catalyst for direct epoxidation of propylene is one of the most important problems in heterogeneous catalysis and surface chemistry, and it is often referred to as the holy grail of heterogeneous catalysis.

Lei et al. recently showed that size-selected clusters of $\mathrm{Ag}$ $\left(\mathrm{Ag}_{3}\right.$ trimers; Figure 1), and small aggregates of the $\mathrm{Ag}$ trimers (effectively nanoparticles with a diameter of ca. $3.5 \mathrm{~nm}$ ) dis-

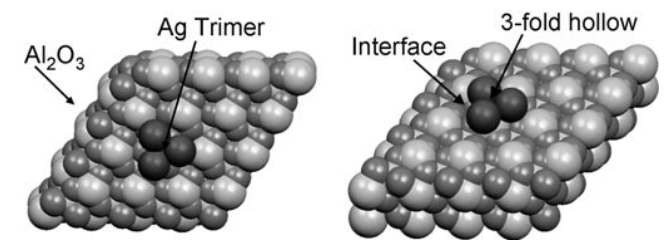

Figure 1. Two different views of a model depicting Ag trimer on alumina. The arrows point to oxygen adsorption sites.

persed on amorphous alumina films exhibit high selectivity in epoxidation of propylene to form PO. ${ }^{[5]}$ The selectivity measurements were performed in temperature-programmed reaction (TPR) studies whereby the performance of the catalysts was measured as a function of the catalyst temperature. The PO selectivity generally decreased at higher temperatures. DFT calculations showed that the pathway to PO included the activation of the $\mathrm{C}=\mathrm{C}$ double bond in propylene leading to the formation of the surface oxametallacycle intermediate, which isomerizes in an elementary step to form PO. The critical advantages that make the trimer and aggregate catalysts more selective than larger Ag particles include their unique electronic structure, which allows them to preferentially activate the $\mathrm{C}=\mathrm{C}$ double bond in propylene (rather than allylic $\mathrm{C}-\mathrm{H}$ bonds) forming oxametallacycles, and their small size, which sterically limits their ability to activate $\mathrm{C}-\mathrm{H}$ bonds in the oxametallacycle intermediate.

The selectivity in catalytic epoxidation of ethylene to form ethylene oxide-an analogous reaction to propylene epoxidation, as it involves the selective addition of atomic oxygen across a $\mathrm{C}=\mathrm{C}$ double bond-was recently shown to also be significantly affected by the shape of catalytic Ag particles. ${ }^{[6,7]}$ 


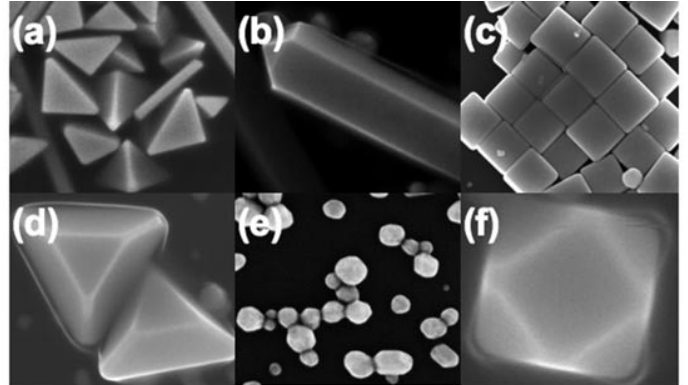

(g)

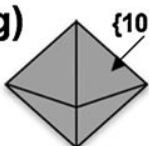

$\{100\}$ (h)

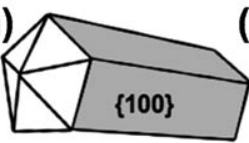

(i)

$\{100\}$
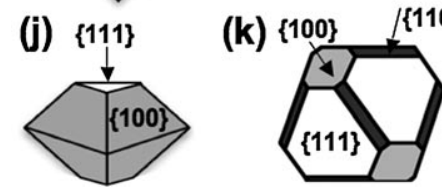

(I)

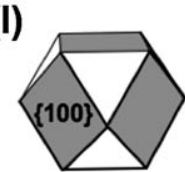

Figure 2. SEM images and structural models of different shapes of Ag metal particles: $a, g$ ) right bipyramids; $b, h$ ) pentagonal nanowires; $c, i)$ cubes; $d, j)$ truncated right bipyramids; e, k) quasi-spherical; $f, l$ ) truncated cubes.

The cubic and pentagonal nanowire shapes (Figure 2) exhibit higher selectivity than conventional spherical particles. The superior selectivity of the cubic and nanowire particles was attributed to the fact that these particles are terminated mainly by the (100) surface facets, rather than the lowest energy (111) facet that dominates spherical particles. DFT calculations showed that the (100) surface facet is inherently more selective to ethylene oxide compared to the (111) facet.

These reports clearly illustrate that the size and shape of catalytic particles represent critical design levers that can be exploited to design catalytic structures with high concentrations of the surface sites that exhibit high selectivity. On a very basic level, by controlling size and shape we are effectively synthesizing surface sites that fill the large gap in the chemical activity of neighboring elements in the periodic table, allowing us to tune and optimize heterogeneous catalysts. This ability to tune chemical reactivity of heterogeneous catalysts by controlled synthesis of targeted sites is particularly important for the design of selective catalysts, because small changes in the inherent chemical reactivity of a site can lead to much larger changes in selectivity toward a particular product. In addition, these reports also suggest that quantum chemical density functional theory (DFT) calculations have reached a level of maturity to be useful not only in explaining various experimental observations, but also in guiding the discovery of optimal catalytic sites. Without useful predictive theories and models, such as those offered by DFT calculations and detailed experimental studies of the mechanisms of relevant surface reactions, it will be impossible to identify the surface sites that exhibit optimal selectivity and that should be targeted in the process of catalyst synthesis.

Although the findings discussed above indicate that heterogeneous catalysis could play a larger role in the commercial synthesis of high-value, low-volume commodity chemicals, there are a number of questions that still need to be addressed. Strategies for the synthesis of shape- and size-selected metal particles are inherently complex and do not lend themselves to convenient scale-up. Furthermore, controlled synthesis of particles of targeted shapes and sizes directly on high surface area supports is currently not possible. In addition, the unique chemical activity of the above-discussed metal nanostructures is derived from their unique geometric and electronic features, which in turn are related to the fact that these structures are not the most thermodynamically stable structures. In general, under reaction conditions there will be a thermodynamic driving force for metal particles of different shapes to collapse into the lowest energy structures, and for small clusters to agglomerate into larger structures. It is imperative to identify approaches to preserve the targeted optimal shape and size of catalytic particles in reactive environments on commercial time scales, which are two to three orders of magnitude larger than standard lab time scales under which the reactions reported in the aforementioned contributions were performed. One possible way to accomplish this task relies on lowering the operating temperature of the process. The low operating temperatures would reduce the frequency of events leading to the changes in the size and shape of catalytic particles. Naturally, this means catalytic sites need to be able to activate chemical transformations at reasonable turnover frequencies at low temperatures. Another possible approach is to increase the inherent stability of the size- and shape-specific structures. It has been suggested that this can be accomplished by encasing these structures in oxide shells or stabilizing them with organic stabilizer molecules adsorbed on the surface of the nanostructures. ${ }^{[8,9]}$ All of these approaches are at embryonic stages of development.

In conclusion, recent developments in the areas of DFT modeling of catalytic reactions, powerful experimental techniques allowing us to study surface chemical transformation at molecular scales and often under relevant conditions, and controlled synthesis of the targeted catalytic sites represent landscape-changing advances that might create opportunities for the design of catalysts for the selective heterogeneous production of low-volume, high-value commodity chemicals. While these advances are encouraging, the scale-up issues in the synthesis of exotic nanostructures and their long-term stability under reacting environments need to be addressed.

\section{Acknowledgements}

We gratefully acknowledge the support of the US Department of Energy DOE-BES, Division of Chemical Sciences (FG-0205ER15686) and NSF (CTS-CAREER 0543067 and CBET 0966700). $S L$ also acknowledges the DuPont Young Professor grant by the DuPont Corporation and the Camille Dreyfus Teach-Scholar Award from the Camille \& Henry Dreyfus Foundation.

Keywords: density functional calculations - epoxidation nanoparticles $\cdot$ selectivity $\cdot$ silver 
[1] A. T. Bell, Science 2003, 299, 1688

[2] J. K. Nørskov, T. Bligaard, J. Rossmeisl, C. H. Christensen, Nat. Chem. 2009, $1,37$.

[3] R. Schlögl, S. B. A. Hamid, Angew. Chem. 2004, 116, 1656; Angew. Chem. Int. Ed. 2004, 43, 1628.

[4] T. Bligaard, J. K. Nørskov, S. Dahl, J. Matthiesen, C. H. Christensen, J. Sehested, J. Catal. 2004, 224, 206.

[5] Y. Lei, F. Mehmood, S. Lee, J. Greeley, B. Lee, S. Seifert, R. E. Winans, J. W. Elam, R. J. Meyer, P. C. Redfern, D. Teschner, R. Schlogl, M. J. Pellin, L. A. Curtiss, S. Vajada, Science 2010, 328, 224.
[6] P. Christopher, S. Linic, J. Am. Chem. Soc. 2008, 130, 11264.

[7] P. Christopher, S. Linic, ChemCatChem 2010, 2, 78.

[8] S. H. Joo, J. Y. Park, C. K. Tsung, Y. Yamada, P. Yang, G. A. Somorjai, Nat. Mater. 2009, 8, 126.

[9] D. Aherne, D. E. Charles, M. E. Brennan-Fournet, J. M. Kelly, Y. K. Gun'ko, Langmuir 2009, 25, 10165.

Received: May 13, 2010

Published online on August 2, 2010 